\title{
Principais fatores que influenciam na adesão do tratamento da Hipertensão Arterial Sistêmica: uma revisão integrativa
}

\author{
Main factors influencing treatment adherence for Systemic Arterial Hypertension: an integrative \\ review
}
Principales factores que influyen en la adherencia al tratamiento para la Hipertensión Arterial Sistémica: una revisión integradora

Recebido: 15/12/2021 | Revisado: 21/12/2021 | Aceito: 28/12/2021 | Publicado: 06/01/2022

Gabriella Farias Batista
ORCID: https://orcid.org/0000-0003-2436-5752
Universidade Ceuma, Brasil
E-mail: gabriellafarias45@gmail.com
Aritana Carvalho de Moura Nascimento
ORCID: https://orcid.org/0000-0002-2431-3066
Universidade Ceuma, Brasil
E-mail: aritana_moura@hotmail.com
Bárbara de Freitas Souza
Eniversidade Ceuma, Brasil
E-mail: barbara_f_freitas@hotmail.com
Lia Santoro Alves Tomé
ORCID: https://orcid.org/000-0002-7319-1480
Universidade Ceuma, Brasil
E-mail: lia_santoro@hotmail.com
ORCID: https://orcid.or/0000-0002-0706-2613
Maria Gabryela Oliveira Costa
ORCID: https://orcid.org/0000-0003-3733-4373
Universidade Ceuma, Brasil
E-mail: mariagaby44@hotmail.com
Jocélia Martins Cavalcante Dantas
ORCID: https://orcid.org/0000-0001-5656-6247
Universidade Ceuma, Brasil
E-mail: joceliadantas@uol.com.br
Rafaela Targino

\section{Resumo}

A hipertensão arterial sistêmica é uma condição clínica multifatorial caracterizada por níveis elevados e sustentados de pressão arterial (PA) $\geq 140 \times 90 \mathrm{mmHg}$, com altas taxas de morbimortalidade. Dessa forma, na intenção de promover uma correta adesão das medidas farmacológicas e de mudanças no estilo de vida, o presente estudo tem como objetivo identificar, na literatura, os principais fatores que interferem no processo de adesão ao tratamento da HAS. Realizou-se uma revisão integrativa nas bases de dados SciELO e Medline foram selecionados 16 artigos, publicados entre 2010 e 2020, utilizando os descritores "Hipertensão Arterial Sistêmica" AND adesão AND tratamento. Os principais fatores, encontrados na literatura, relacionados a não adesão ao tratamento anti-hipertensivo foram: sexo masculino, escolaridade baixa, baixa frequência nas consultas da UBS e vinculo frágil com a estratégia de saúde. Infere-se, portanto, a necessidade de conhecer os principais obstáculos para a adesão integral do tratamento visando propor medidas que diminuam a não adesão ao tratamento.

Palavras-chave: Adesão; Tratamento; Hipertensão arterial sistêmica.

\section{Abstract}

Systemic arterial hypertension is a multifactorial clinical condition characterized by high and sustained levels of blood pressure (BP) $\geq 140 \times 90 \mathrm{mmHg}$, with high rates of morbidity and mortality. Thus, in order to promote correct adherence to pharmacological measures and changes in lifestyle, the present study aims to identify, in the literature, the main factors that interfere in the process of adherence to treatment for SAH. An integrative review was carried out in the SciELO and Medline databases 16 articles were selected, published between 2010 and 2020, using the descriptors "Systemic Arterial Hypertension" AND adherence AND treatment. The main factors found in the literature related to 
non-adherence to antihypertensive treatment were: male gender, low education level, low attendance at the UBS and a weak link with the health strategy. It is inferred, therefore, the need to know the main obstacles to full adherence to treatment, aiming to propose measures to reduce non-adherence to treatment.

Keywords: Accession; Treatment; Systemic arterial hypertension.

\section{Resumen}

La hipertensión arterial sistémica es una condición clínica multifactorial caracterizada por niveles altos y sostenidos de presión arterial (PA) $\geq 140$ x $90 \mathrm{mmHg}$, con altas tasas de morbilidad y mortalidad. Así, con el fin de promover la correcta adherencia a las medidas farmacológicas y cambios en el estilo de vida, el presente estudio tiene como objetivo identificar, en la literatura, los principales factores que interfieren en el proceso de adherencia al tratamiento de la HAS. Se realizó una revisión integradora en las bases de datos SciELO y Medline se seleccionaron 16 artículos, publicados entre 2010 y 2020, utilizando los descriptores "Hipertensión Arterial Sistémica" Y adherencia Y tratamiento. Los principales factores encontrados en la literatura relacionados con la no adherencia al tratamiento antihipertensivo fueron: sexo masculino, bajo nivel educativo, baja asistencia a la UBS y un vínculo débil con la estrategia de salud. Se infiere, por tanto, la necesidad de conocer los principales obstáculos a la plena adherencia al tratamiento con el fin de proponer medidas para reducir la no adherencia al tratamiento.

Palabras clave: Adhesión; Tratamiento; Hipertensión arterial sistémica.

\section{Introdução}

A Hipertensão Arterial Sistêmica (HAS) é uma doença crônica não transmissível caracterizada por níveis elevados e sustentados de pressão arterial $(\mathrm{PA}) \geq 140$ x 90mmHg. A qual deve ser medida com a técnica correta, em pelo menos duas ocasiões diferentes, na ausência de medicação anti-hipertensiva. (Cardiologia, 2020). Trata-se de uma condição multifatorial, que relaciona fatores genéticos, epigenéticos, ambientais e sociais (Cardiologia, 2020).

A HAS é uma das doenças mais prevalentes na população mundial, no Brasil, segundo a Pesquisa Nacional de Saúde de 2013, tem prevalência estimada em 32,3\%, chegando a 71,7\% para os indivíduos acima de 70 anos (Cardiologia, 2020). O aumento a pressão arterial (PA) contribuiu direta ou indiretamente em 50\% das mortes por doença cardiovascular (Cardiologia, 2016; Cardiologia, 2020).

O objetivo do tratamento anti-hipertensivo é a manutenção de níveis pressóricos controlados, reduzindo o risco de doenças cardiovasculares, diminuindo a morbimortalidade e melhorando a qualidade de vida dos indivíduos (Brasil, 2013). Portanto, o cuidado do paciente com HAS deve ser multiprofissional e segue duas vertentes de tratamento, o não medicamentoso, (alterações no estilo de vida), e o medicamentoso com fármacos que visem diminuir a pressão arterial (Cardiologia, 2020; Jardim et al, 2017).

Devido ao seu caráter assintomático na maioria dos casos, a HAS está frequentemente associada às alterações funcionais e/ou estruturais de órgãos-alvo como coração, cérebro, rins e vasos. Ela é o principal fator de risco modificável com associação independente, linear e contínua para doenças cardiovasculares, doença renal crônica e morte prematura (Cardiologia, 2020). As lesões subclínicas dos órgãos-alvo são indicadores de gravidade clínica do paciente (Rocha, 1998; Okai, 2013), influenciando o prognóstico dessa população, pelo aumento da morbimortalidade (Okai, 2013). O não controle pressórico decorre na maioria das vezes da baixa adesão do paciente ao tratamento e/ou não conformidade ao seguimento clínico (Zeller, 2007).

Avaliar a adesão ao tratamento antihipertensivo é de vital importância, para que o controle da PA seja efetivamente atingido, uma vez que a baixa aceitação ao tratamento é um dos principais fatores para que seus valores permanecem elevados (Mancia et al., 2014). O presente estudo tem como objetivo levantar na literatura os principais fatores que interferem no processo de adesão ao tratamento da HAS. Dessarte, com a intenção de implementar rotinas que promovam uma correta adesão às medidas farmacológicas e de mudanças no estilo de vida.

\section{Metodologia}

Elegeu-se a revisão integrativa como método de estudo, pois esta proporciona a síntese de conhecimento e a 
incorporação da aplicabilidade de resultados de estudos significativos na prática (Souza et al., 2010). Além disso, trata-se uma abordagem metodológica que permite a inclusão de estudos com diferentes metodologias para a compressão do mesmo fenômeno, permitindo uma visão mais completa e consistente do assunto analisado.

Para a elaboração de uma revisão integrativa, inicialmente os pesquisadores delimitam um objetivo para o estudo e elaboram uma pergunta norteadora, a qual irá guiar a busca na literatura, a fim de responder os questionamentos levantados. A coleta de dados deve obedecer aos critérios de inclusão e exclusão previamente estabelecidos (Souza et al.,2010). Deste modo, para orientar o presente estudo, desenvolve-se a seguinte pergunta: quais são os principais fatores, descritos na literatura, que influenciam na adesão ou não do tratamento da HAS?

O levantamento bibliográfico, realizado no último semestre de 2021, a partir das bases de dados Scientific Electronic Library Online (SciELO) e Medical Literature Analysis and Retrieval Sistem on-line (Medline). Os termos utilizados na busca estão de acordo com os Descritores em Ciências da Saúde (DeCS), da Biblioteca Virtual em Saúde. Sendo utilizados os seguintes descritores "Hipertensão Arterial Sistêmica" AND adesão AND tratamento.

Foram incluídos artigos publicados no ano de 2010 a 2020, nos idiomas português e inglês, disponíveis na integra nas duas bases de dados indicadas e estritamente voltados para o objetivo citado.

Em seguida, após a leitura dos artigos completos foram selecionados 16 artigos. Apresenta-se a seguir um fluxograma do processo de seleção dos artigos para revisão (Figura 1). 
Figura 1. Fluxo do processo de seleção dos artigos para revisão.
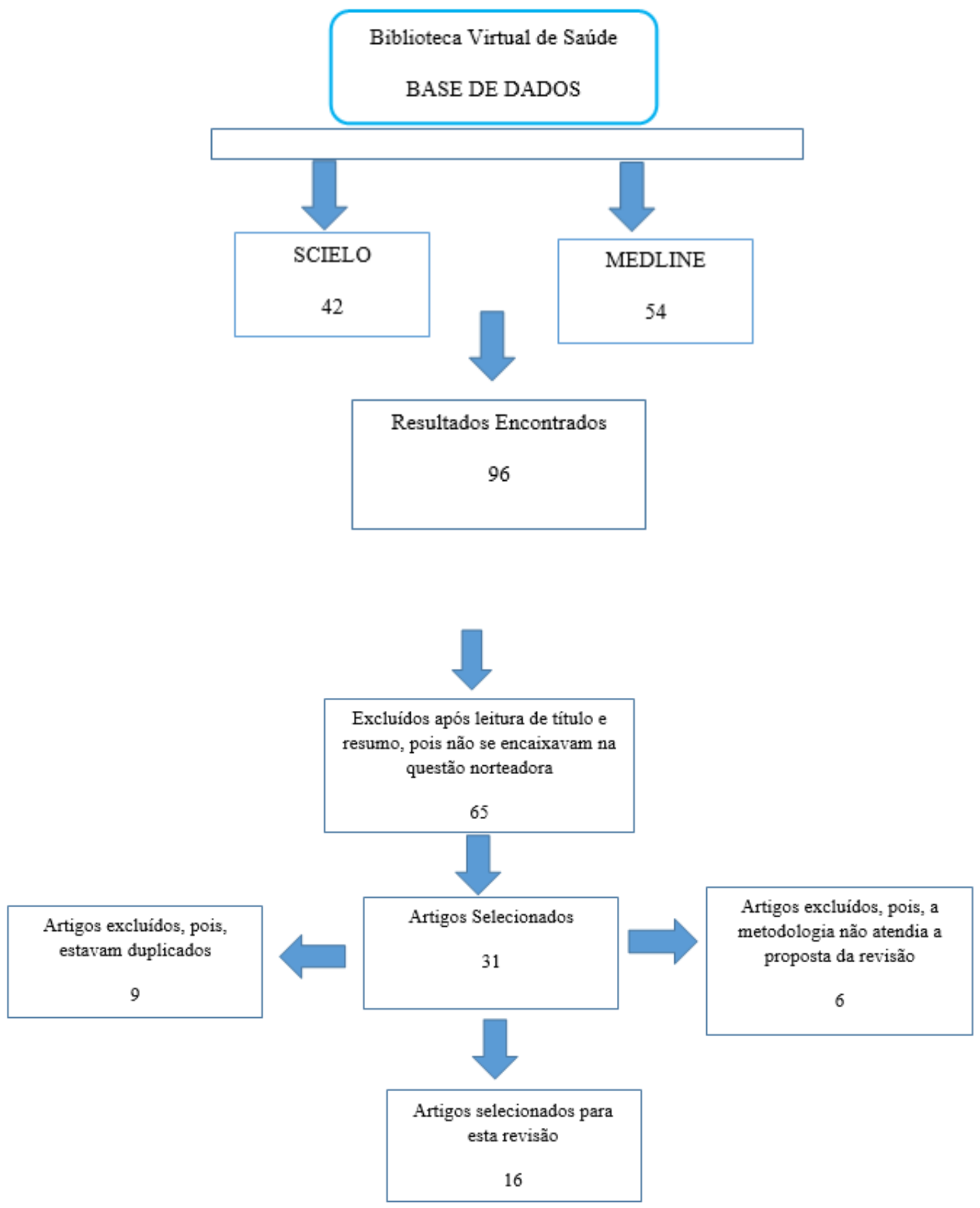

Fonte: Autores.

\section{Resultados e Discussão}

Foram considerados elegíveis 16 artigos em conformidade com a questão norteadora supracitada.

Apresenta-se a seguir um quadro síntese dos artigos selecionados, segundo autor, ano, revista e delineamento de pesquisa. (Quadro 1). 
Quadro 1 - Sumarização dos artigos encontrados.

\begin{tabular}{|c|c|c|c|}
\hline AUTOR & $\begin{array}{c}\text { ANO DE } \\
\text { PUBLICAÇÃO } \\
\end{array}$ & REVISTA & METODOLOGIA \\
\hline Albuquerque et al. & 2018 & Rev Bras Enferm [Internet]. & Estudo analítico \\
\hline Barreto et al. & 2018 & Ciência \& Saúde Coletiva & Estudo transversal analítico \\
\hline Rêgo et al. & 2018 & Rev Bras Enferm [Internet]. & Estudo transversal \\
\hline Jesus et al. & 2016 & Arq Bras Cardiol. & Estudo transversal \\
\hline Machado et al. & 2016 & Rev. Nutr. & $\begin{array}{c}\text { Estudo de intervenção longitudinal, do } \\
\text { tipo ensaio comunitário, comparativo, } \\
\text { de abordagem quantitativa }\end{array}$ \\
\hline Mansour et.al & 2016 & $\begin{array}{c}\text { Epidemiologia e Serviços de } \\
\text { Saúde }\end{array}$ & Estudo transversal \\
\hline Tavares et al. & 2016 & Rev Bras Enferm [Internet]. & Estudo transversal \\
\hline Souza et al. & 2016 & Revista de Saúde Pública & Revisão Sistematica com metaanalise \\
\hline Magnabosco et al. & 2015 & $\begin{array}{l}\text { Rev. Latino-Am. } \\
\text { Enfermagem }\end{array}$ & $\begin{array}{c}\text { Estudo analítico baseado em inquérito } \\
\text { epidemiológico }\end{array}$ \\
\hline $\begin{array}{l}\text { Vancini-Campanharo } \\
\text { et al. }\end{array}$ & 2015 & $\begin{array}{l}\text { Rev. Latino-Am. } \\
\text { Enfermagem }\end{array}$ & Estudo transversal \\
\hline Martins et al. & 2014 & Acta Paul Enferm. & Estudo transversal \\
\hline Eid et al. & 2013 & Rev. Eletr. Enf. [Internet]. & $\begin{array}{l}\text { Estudo descritivo com abordagem } \\
\text { quantitativa }\end{array}$ \\
\hline Leão e Silva et al. & 2013 & $\begin{array}{l}\text { Physis Revista de Saúde } \\
\text { Coletiva } \\
\end{array}$ & ---- \\
\hline Grezzana et al. & 2012 & Arq Bras Cardiol & Estudo transversal \\
\hline Lima et al. & 2010 & Rev. Rene. & Estudo exploratório-descritivo \\
\hline Santos et al. & 2010 & $\begin{array}{c}\text { Brazilian Journal of } \\
\text { Pharmaceutical Sciences }\end{array}$ & $\begin{array}{c}\text { Estudo transversal, descritivo, } \\
\text { observacional com abordagem } \\
\text { quantitativa }\end{array}$ \\
\hline
\end{tabular}

Fonte: Autores.

A adesão ao tratamento pode ser definida, de acordo com a Organização Mundial da Saúde, como o ato do paciente de realizar as tomadas das medicações, seguir dieta e/ou possuir hábitos de vida saudáveis de acordo com o prescrito pelo profissional de saúde. Portanto, a adesão ao tratamento é considerada um processo multifatorial e complexo, o qual leva em consideração fatores ambientais, psicossociais e individuais (Albuquerque et. al, 2018).

Em um estudo realizado por Tavares et.al (2016) com 1029 idosos da cidade de Uberaba-MG, 524 (50,9\%) aderiam ao tratamento farmacológico para HAS e 505 (49,1\%) não aderiam ao tratamento. Houve maior proporção de idosos mais velhos entre os aderentes ao tratamento farmacológico (22,3\%) quando comparados aos não aderentes (16,8\%). Os idosos não aderentes ao tratamento farmacológico apresentaram maior número de morbidades do que os aderentes, corroborando com os achados do estudo realizado por Albuquerque et.al (2018) no qual mostrou que os não aderentes ao tratamento têm maior probabilidade de internações nos serviços de emergência devido a complicações cardiovasculares e cerebrovasculares quando comparados aos que aderem ao tratamento. Evidenciando dessa forma, um fator agravante da não adesão ao tratamento da HAS.

Ainda sobre pesquisas envolvendo idosos Leão e Silva et.al (2013), realizaram um estudo que objetivou identificar as percepções de pacientes idosos hipertensos em torno da terapêutica, relacionadas à adesão ou não ao tratamento medicamentoso. Constatou-se que 62,6\% dos idosos não aderem ao tratamento anti-hipertensivo prescrito pela equipe médica por considerarem a HAS como uma doença aguda, sintomática e ligada a estados emocionais. Em relação aos fatores envolvidos à não adesão os 
sociodemográficos foram os mais importantes como, classe socioeconômica mais baixa e maior proporção de indivíduos de raça negra.

Magnabosco et.al (2015) elencaram os fatores associados à não adesão ao tratamento medicamentoso da hipertensão arterial sistêmica: gênero masculino, faixa etária entre 20 e 59 anos, baixa classe econômica, etilismo, tempo curto de diagnóstico de hipertensão e não procura pelo serviço de saúde para consultas de rotina, corroborando com os resultados do estudo de Leão e Silva et.al (2013) mesmo sendo realizado em populações diferentes.

Porém um estudo realizado por Eid et al. (2013) com 90 hipertensos, destacam fatores contrários ao elencado acima, como: a maioria era do sexo feminino (62,2\%), brancos (86,7\%), com idade na faixa de 34 a 84 anos, casados $(66,7 \%)$. As ocupações mais frequentes foram aposentado-pensionistas $(72,2 \%)$ e do lar $(16,7 \%), 82(91,1 \%)$ com renda familiar de um a três salários mínimos, com dois ou mais dependentes $(57,8 \%)$.

É importante ressaltar que o estudo realizado por Tavares et.al (2016) não identificou associação entre sexo e estado civil com a aderência ao tratamento farmacológico, divergindo da maioria da literatura científica, a qual aponta a família, muitas vezes representada pela figura do cônjuge, como parte fundamental na adesão ao tratamento farmacológico.

Outro fator que interfere na adesão terapêutica, encontrada durante a realização desta revisão, foi o vínculo entre a equipe de saúde e o paciente. O estudo transversal publicado por Rêgo et. al (2018) com 417 pessoas em tratamento da hipertensão arterial avaliou a satisfação do usuário hipertensos com os serviços prestados pela Unidade Básica de Saúde (UBS) associado as características clínicas e os atributos referentes à UBS. Este constatou que os pacientes com controle pressórico inadequado possuem mais chances de avaliar a orientação do profissional da unidade de saúde sobre a importância de seguir o tratamento indicado para o controle da HA como insatisfatório. Além de salientar a falta de orientação por parte do profissional medico como um fator que dificulta o controle pressórico.

Estudo transversal analítico conduzido por Barreto et. al (2018) verificou a adesão à farmacoterapia pelo Questionário de Não-adesão de Medicamentos da Equipe Qualiaids (QAM-Q), desenvolvido para abordar o ato, o processo e o resultado da adesão, PA controlada ou não. O total de entrevistados foram 422 dos quais $47(11,1 \%)$ relataram não ter utilizado, mediante procura espontânea, consultas médicas disponíveis na Atenção Básica (AB) nos últimos seis meses. Ademais, foi possível verificar que há maiores chances de não adesão ao tratamento medicamentoso e descontrole pressórico entre os indivíduos que não utilizaram as consultas de rotina disponíveis na $\mathrm{AB}$.

O maior comparecimento a consultas está relacionado com a melhor adesão ao tratamento, o que ampara a relação entre o acesso e a utilização dos serviços de saúde com o melhor acompanhamento dos pacientes hipertensos com comorbidades cardiovasculares associadas (Albuquerque et. al, 2018).

A associação entre o nível de informação obtida pelo paciente e a sua adesão ou não ao tratamento foi verificada no estudo realizado por Martins et al. (2014), o qual identificou que 81,4\% dos estudados mencionaram ter obtido orientações sobre dieta, atividade física, tabagismo e ingesta de bebida alcoólica. Esses sinalizaram mudanças nos seus hábitos de vida após tais orientações.

A revisão sistemática realizada por Souza et.al (2016) cujo objetivo era verificar os efeitos do tratamento antihipertensivo, farmacológico e não-farmacológico, na qualidade de vida relacionada à saúde de pessoas com hipertensão demostrou que o tratamento não farmacológico melhora a qualidade de vida global e o domínio físico de pessoas com HAS, impactando positivamente nos domínios mental, físico e escore total da Qualidade de Vida Relacionada a Saúde.

Ademais, o esquema terapêutico também foi relacionado como um possível obstáculo para a adesão ao tratamento, seja pelo esquecimento, pelo incomodo de tomar vários comprimidos ou pelo efeito adverso destes. Quanto maior a quantidade de medicamentos utilizados, maior o risco de interações e reações adversas, diminuindo, dessa forma, a adesão ao tratamento e, por consequência, um pior controle da PA (Jesus et al., 2016). Outra barreira à adesão ao tratamento farmacológico é a recordação 
em relação ao uso do medicamento, principalmente naqueles em que se usa mais de um fármaco, sugerindo-se que a monoterapia seja uma opção para facilitar a adesão ao tratamento e, em casos em que isso não é possível, é necessário que a equipe busque estratégias juntamente com o paciente para diminuir a taxa de esquecimento (Vancini-Campanharo et.al, 2015).

Santos et.al (2010) realizaram um estudo que teve como objetivo avaliar os níveis de pressão arterial, assim como o nível de adesão ao tratamento farmacológico com maleato de enalapril de pacientes portadores de hipertensão arterial, usuários de uma Farmácia Escola. Dos 102 pacientes entrevistados, 65,7\% apresentaram níveis pressóricos controlados, porém apenas 36,3\% indicaram níveis de adesão total à terapêutica farmacológica. Grezzana et.al (2012) elaboraram um estudo semelhante cujo objetivo era estimar a associação entre a adesão ao tratamento farmacológico anti-hipertensivo e o controle das medidas de PA realizadas pela monitoração ambulatorial de 24 horas, em pacientes hipertensos cadastrados no Programa Hiperdia de unidades básicas de saúde (UBS) de um pequeno município do sul do Brasil. Obtiveram como resposta que do total de 143 pacientes avaliados, 55,2\% foram identificados como HAS controlada $(<130 / 80 \mathrm{mmHg}$ ) pelas medidas da MAPA de 24 horas, 44,8\% não estavam controlados e quando avaliados em relação a adesão ao tratamento medicamentoso, $65,7 \%$ da amostra foram consideradas aderentes ao tratamento proposto, enquanto $20,3 \%$ eram moderadamente aderentes e $14 \%$ foram classificados como não aderentes.

Em um estudo exploratório-descritivo, Lima et.al (2010) observaram que as medidas aderidas foram as seguintes: consumo adequado de sal e de café, abstenção do álcool e do tabaco, ingestão de gordura vegetal, preferência por vegetais e carnes brancas, gerenciamento do estresse e uso regular do medicamento. Porém na adesão ao tratamento não havia relação entre saber ou não acerca da HAS mas sim em uma relação automática sobre o que o profissional de saúde orienta ou não. Ficando então uma reflexão sobre processo de educação permanente, capaz de conduzir a pessoa hipertensa à adesão às condutas de controle da HAS de forma mais consciente. Machado et.al (2016) evidenciaram que que as intervenções educacionais aumentam a adesão ao tratamento não farmacológico da hipertensão.

Outro fator bem consolidado na literatura cientifica que atrapalha a adesão de maneira satisfatória é o atual modelo de saúde mais focado no tratamento de doenças que na prevenção e em educação e saúde. Além disso, o modelo de saúde voltada para o tratamento da doença sobreposto a prevenção é demasiadamente oneroso. No Brasil, anualmente, o sistema de saúde contabiliza, em média, um milhão de internações por doenças do aparelho circulatório, com um custo aproximado de um bilhão e 800 milhões de reais (Barreto et al, 2018). Contrapartida quando o investimento é voltado para atividades preventivas e promotoras da saúde, como por exemplo palestras, campanhas educativas e consultas programadas e quando a população tem acesso adequado aos serviços básicos de saúde reduz-se consideravelmente o número de hospitalizações por condições sensíveis à Atenção Básica, entre elas os agravos das condições crônicas. Mansour et al (2016) afirmam que em situação de adequada assistência fatores relacionados aos aspectos individuais perdem influência na determinação da não adesão, sendo superados pela oferta efetiva de atenção à saúde e pleno acesso a medicamentos.

\section{Conclusão}

A realização desse artigo foi de suma importância para o conhecimento dos principais fatores que interferem na adesão ou não do tratamento anti-hipertensivo das pessoas que são portadoras de Hipertensão Arterial Sistêmica, sendo um processo complexo que envolve fatores ambientais, emocionais e sociais.

Destaca-se como principais fatores: as condições socioeconômicas (classe social mais baixa), vínculo entre a equipe de saúde e o paciente, o comparecimento as consultas, a quantidade de medicamentos prescritas, a realização de atividade educativas.

Por outro lado, é importante destacar algumas limitações do presente estudo, como por exemplo, a população descrita é em sua maioria atendida pelo SUS. Sendo poucos os achados na literatura que descrevem a adesão ao tratamento anti- 
hipertensivo da população que faz uso de consultas particulares, fazendo necessários a realização de estudos nesse cenário.

\section{Referências}

Albuquerque, N. L. S., Oliveira, A. S. S., Silva, J. M. \& Araújo, T. L. (2018). Association between follow-up in health services and antihypertensive medication adherence. Rev Bras Enferm

Brasil. (2013). Ministério da Saúde. Secretaria de Atenção à Saúde. Departamento de Atenção Básica Saúde do adulto: Hipertensão Arterial Sistêmica. Cadernos de atenção básica. Ed. Ministério da Saúde.

Barreto, M. S., Mendonça, R. D., Pimenta, A. M., Garcia-Vivar, C. \& Marcon, S. S. (2018). Não utilização de consultas de rotina na Atenção Básica por pessoas com hipertensão arterial. Ciência \& Saúde Coletiva. 23, 795-804.

Barroso, W. K. S. (2021). Diretrizes Brasileiras de Hipertensão Arterial-2020. Arquivos Brasileiros de Cardiologia. 116, 516-658

Eid, L. P., Nogueira, M. S., Veiga, E. V., Cesarino, E. J. \& Alves, L. M. M. (2013). Adesão ao tratamento anti-hipertensivo: análise pelo Teste de MoriskyGreen. Revista Eletrônica de Enfermagem, 15(2), 362-7.

Grezzana, G. B., Stein, A. T. \& Pellanda L. C. (2013) Adesão ao tratamento anti-hipertensivo em atenção primária. Arq Bras Cardiol. 100(4):355-361.

Jardim, L. M. S. S. V., Jardim, T. V., Souza, W. K. S. B., Pimenta, C. D., Sousa, A. L. L. \& Jardim, P. C. B. V. (2017). Tratamento multiprofissional da hipertensão arterial sistêmica em pacientes muito idosos. Arq Bras Cardiol., 108, 53-59.

Jesus, N. S., Nogueira, A. R., Pachu, C. O., Luiz, R. R. \& Oliveira, G. M. M. (2016). Adesão ao tratamento e Controle da Pressão Arterial após participação no ReHOT. Arquivos Brasileiros de Cardiologia, 107, 437-445.

Leão e Silva, L. O., Soares, M. M., Oliveira, M. A., Rodrigues, S. M., Machado, C. J. \& Dias, C. A. (2013) "Tô sentindo nada”: percepções de pacientes idosos sobre o tratamento da hipertensão arterial sistêmica. Physis Revista de Saúde Coletiva, 23: 227-242.

Lima, H. P., Santos, Z. M. S. A., Nascimento, J. C. \& Caetano, J. Á. (2010). Adesão do usuário hipertenso ao tratamento e a interface com o saber sobre o agravo. Rev. Rene. 11(2), 70-178.

Machado, J C., Cotta, R M. M., Moreira, T. R. \& Silva, L. S. (2016) Adherence to non-pharmacological treatment: Analysis of the impact of three health educational and nutritional strategies in hypertensive patients. Rev. Nutr., 29(1):11-22.

Magnabosco, P., Teraoka, E. C., Oliveira, E. M., Felipe, E. A., Freitas, D. \& Marchi-Alves, L. M. (2015) Análise comparativa da não adesão ao tratamento medicamentoso da hipertensão arterial sistêmica em população urbana e rural. Rev. Latino-Am. Enfermagem. 23(1):20-7.

Malachias, M. V. B. (2016) $7^{\text {a }}$ Diretriz Brasileira de Hipertensão Arterial: Capítulo 1-Conceituação, Epidemiologia e Prevenção Primária. Arquivos Brasileiros de Cardiologia, 107, 1-6.

Mansour, S. N., Monteiro, C. N. \& Luiz, O. C. (2016). Adesão ao tratamento farmacológico de pacientes hipertensos entre participantes do Programa Remédio em Casa. Epidemiologia e Serviços de Saúde, 25, 647-654.

Mancia, G. (2014) Guidelines da ESH/ESC para o Tratamento da Hipertensão Arteriale. Rev Port Hipertens e Risco Cardiovasc, 39.

Martins, A. G., et.al. (2014). Adesão ao tratamento clínico ambulatorial da hipertensão arterial sistêmica. Acta Paul Enferm.; 27(3):266-72.

Rêgo A. S, \& Radovanovic C. A. T. (2018) Adherence of hypertension patients in the Brazil's Family Health Strategy. Rev Bras Enferm 71(3):1030-7. http://dx.doi.org/10.1590/0034-7167-2017-0297.

Rocha, E. et al. (1998). Prevalência das lesões nos órgãos-alvo associadas à hipertensão: significado clínico-epidemiológico do predomínio da pressão arterial sistólica. Revista da Faculdade de Medicina de Lisboa, 175-194.

Santos, B. R. M. et.al. (2010). Blood pressure levels and adherence to treatment of hypertensive patients, users of a school pharmacy. Brazilian Journal of Pharmaceutical Sciences 46(3).

Souza, A. C. C. de; et al. (2016). Qualidade de vida e adesão ao tratamento em hipertensão: revisão sistemática com metanálise. Revista de Saúde Pública, 50, 71.

Souza, M. T. de; Silva, M. D. da; \& Carvalho, R. de. (2010). Revisão integrativa: o que é e como fazer. Einstein. 8, 102-106.

Okai, H. (2013). Prevalência de lesões subclínicas de órgãos-alvo numa população hipertensa. Revista Portuguesa de Medicina Geral e Familiar, 29(4), 258261.

Tavares, D. M. dos S. et al. (2016). Qualidade de vida e adesão ao tratamento farmacológico entre idosos hipertensos. Revista Brasileira de Enfermagem, 69, 134-141.

Vancini-Campanharo, C. R. et.al. (2015). Hipertensão Arterial Sistêmica no Serviço de Emergência: adesão medicamentosa e conhecimento da doença. Rev. Latino-Am. Enfermagem nov.-dez.;23(6):1149-56.

Zeller, C. B. et al. (2007). Hipertensão arterial e órgão-alvo: a importância do tratamento. Revista Brasileira de Hipertensão, 14(1), 60-62. 